\title{
Fluid flow induced by nonuniform ac electric fields in electrolytes on microelectrodes. I. Experimental measurements
}

\author{
N. G. Green, ${ }^{1}$ A. Ramos, ${ }^{1}$ A. González, ${ }^{2}$ H. Morgan, ${ }^{3}$ and A. Castellanos ${ }^{1}$ \\ ${ }^{1}$ Departamento de Electrónica y Electromagnetismo, Facultad de Física, Universidad de Sevilla, Reina Mercedes s/n, \\ 41012 Sevilla, Spain \\ ${ }^{2}$ Departamento de Física Aplicada, ESI Universidad de Sevilla, Camino de Los Descubrimientos s/n, 41092 Sevilla, Spain \\ ${ }^{3}$ Bioelectronics Research Centre, Department of Electronics and Electrical Engineering, University of Glasgow, \\ Oakfield Avenue, Glasgow G12 8LT, Scotland, United Kingdom
}

(Received 12 August 1999)

\begin{abstract}
Under the influence of an ac electric field, electrolytes on planar microelectrodes exhibit fluid flow. The nonuniform electric field generated by the electrodes interacts with the suspending fluid through a number of mechanisms, giving rise to body forces and fluid flow. This paper presents the detailed experimental measurements of the velocity of fluid flow on microelectrodes at frequencies below the charge relaxation frequency of the electrolyte. The velocity of latex tracer particles was measured as a function of applied signal frequency and potential, electrolyte conductivity, and position on the electrode surface. The data are discussed in terms of a linear model of ac electroosmosis: the interaction of the nonuniform ac field and the induced electrical double layer.

PACS number(s): 82.70.Dd, 47.65.+a, 82.45.+z, 85.90.+h
\end{abstract}

\section{INTRODUCTION}

The ac electrokinetic manipulation of particles is rapidly becoming a major area of research, with particular applications in biotechnology. The techniques employed in this field allow characterization, identification, and separation of different particle types in solution quickly and efficiently [1-3]. Recently, these methods have been applied to the submicrometer scale for the characterization and separation of latex spheres [4-6], viruses [7-9], DNA, and proteins [10]. The electric fields are applied to particles in solution by microelectrodes fabricated using semiconductor-manufacturing processes [11]. The high electric field strengths can induce fluid flow, and such flows have been observed in many ac electrokinetic experiments $[9,12]$.

This paper describes a recently identified fluid flow which is observed at low frequencies (below $\sim 1 \mathrm{MHz}$ ) [12]. On coplanar electrodes, the direction of this flow is always perpendicular to the electrode edge, driving fluid onto the electrode surface. This type of flow has been used for the separation of submicrometer particles [13]. It has been demonstrated that the fluid flow cannot be explained by electrothermal mechanisms [12]; instead the mechanism of electroosmosis has been postulated as the origin $[14,15]$. Electroosmotic flow arises from the force exerted on the electrical double layer by a tangential electric field [16]. In the planar microelectrode arrays used for ac electrokinetics, divergent electric fields are generated, and as a result a component of the electric field lies tangential to the electrical double layer which is induced on the electrode surface. Therefore, ions in the diffuse double layer experience a force that has a time average that acts from the edge, across the surface of the electrode. This mechanism has been termed ac electroosmosis $[14,15]$.

This paper presents details of the fluid velocity measured using titanium microelectrodes, over a wide range of experimental conditions. The velocity data are presented as a func- tion of applied signal frequency, potential, electrolyte conductivity, and distance from the electrode edge. In order to characterize the frequency variation of the double layer, the impedance of the microelectrodes was measured. Previous observations $[14,15]$ have indicated the importance of electrode polarization [17] in the mechanism responsible for the fluid flow. The results are compared to a circuit model which qualitatively describes the ac electro-osmotic flow [15].

\section{EXPERIMENTAL METHOD}

Measurements of fluid motion were made on microelectrode structures fabricated using microelectronic manufacturing techniques. Pairs of plate electrodes were fabricated on a planar glass substrate (microscope slide) using photolithography [11], consisting of layers of 10-nm titanium, 100-nm gold, and 20-nm titanium. The purpose of the top layer of titanium was to reduce the effects of corrosion at low frequencies and high potentials. The electrodes were $2 \mathrm{~mm}$ long and $100 \mu \mathrm{m}$ wide, and the gap between the electrodes was $25 \mu \mathrm{m}$. A photograph of an electrode array is shown in Fig. 1(a), with a schematic diagram in Fig. 1(b).

ac signals were provided by a function generator (Thurlby Thandar Inst. TG120, RS components, UK) and the voltage and frequency measured independently using a digital oscilloscope (Hewlett-Packard, USA). Three different concentrations of $\mathrm{KCl}$ in water were used for the electrolytes with conductivities measured using a Hewlett-Packard impedance analyzer HP4192A and a conductivity cell (Semtec, UK). The impedance of the electrodes when covered in electrolyte was also measured using the impedance analyzer. Submicrometer fluorescently loaded latex spheres of two different sizes, 557- and 216-nm diameters, were used as tracer particles to measure fluid velocity. Particle movement was observed using a fluorescence microscope (Nikon Microphot), and recorded using a digital camera and $S$-VHS video (JVC). The video was transferred to a computer and analyzed frame 


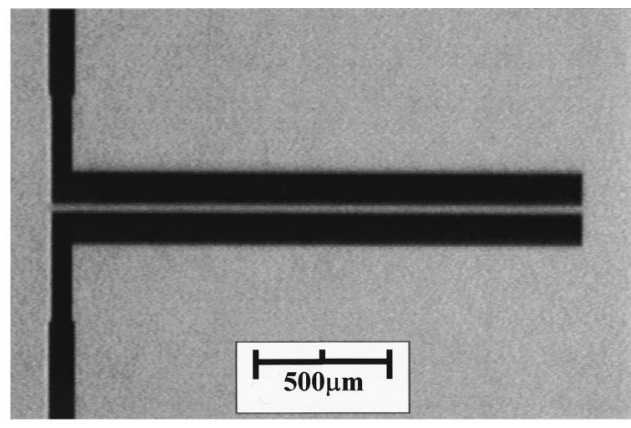

(a)

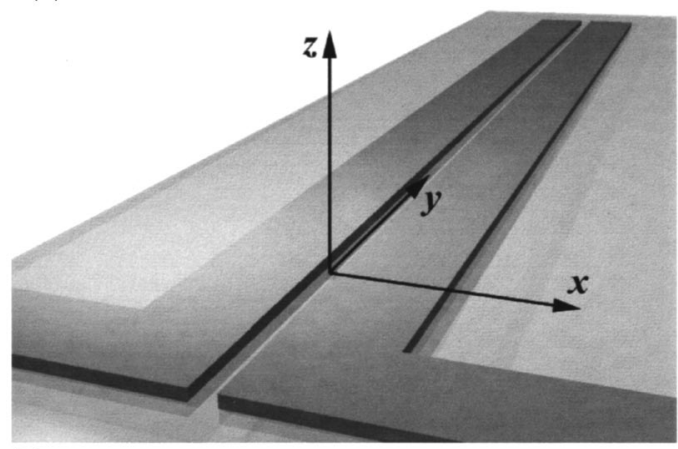

(b)

FIG. 1. (a) A photograph of the coplanar plate microelectrode array used in this paper. The two electrodes are $2 \mathrm{~mm}$ long and 100 $\mu \mathrm{m}$ wide, and separated by a $25-\mu \mathrm{m}$ gap. (b) A schematic diagram of the coplanar plate microelectrode array showing the definition of the axes used in the theory.

by frame to give data in the form of individual particle position as a function of time.

\section{RESULTS}

\section{A. Impedance measurements}

For ease of reference, the three solutions of $\mathrm{KCl}$ will be referred to as $A, B$, and $C$. The conductivities of the electrolytes were measured (at $20^{\circ} \mathrm{C}$ ) as $A: 2.1 \times 10^{-3} \mathrm{Sm}^{-1} ; B$ : $8.6 \times 10^{-3} \mathrm{Sm}^{-1}$; and $C: 8.4 \times 10^{-2} \mathrm{Sm}^{-1}$. In order to characterize the polarization of the electrodes, and therefore the variation in potential dropped across the double layer and the electrolyte, the impedance of the electrode array when covered with electrolyte was measured as a function of frequency. Taking the total impedance of the system to be a capacitor and resistor in parallel, the capacitance and the conductance were determined as a function of frequency. The results are shown in Fig. 2. The limiting values at high frequencies correspond to the capacitance and conductance of the bulk electrolyte, since double-layer effects from the electrode-fluid interface are not present at these frequencies. Deviation from the bulk values at lower frequencies is due to the polarization of the electrodes [17].

\section{B. Observed particle movement}

Movement of the two different sizes of particles was observed simultaneously over a range of applied voltages and frequencies in each electrolyte. A schematic diagram of the observed particle trajectories for a fixed voltage is shown in Fig. 3(a). At high frequencies, movement was only observed (a)

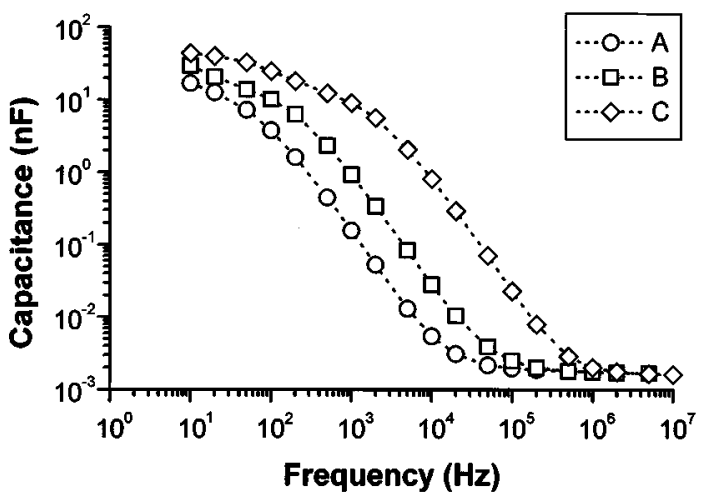

(b)

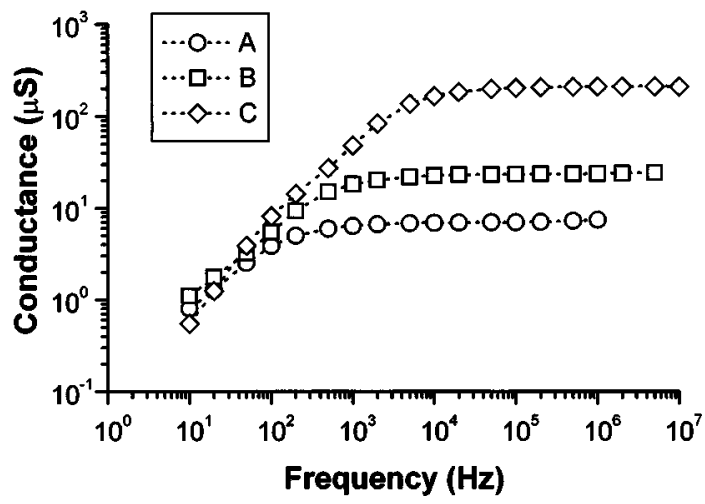

FIG. 2. The measured impedance of the electrode array covered by the three electrolytes, plotted as a function of frequency. The different curves for the three electrolytes are marked as shown in the figures, with the measured capacitance shown in (a) and the measured conductance shown in (b).

close to the electrode edge, and particles collected in a band on the electrode surface before dispersing much more slowly under the influence of diffusion. As the applied frequency was decreased, the magnitude of the particle velocity rose to a maximum and the particles moved across the entire electrode surface and out of the field of observation. As the frequency was decreased further, the magnitude of the particle motion decreased, but particles continued to move across the entire electrode surface in the field of observation. A sequence of experimental images of this variation in behavior can be found in Ref. [12]. The frequency variation in the velocity did not appear to depend on voltage, but was different in the different electrolytes, while the magnitude of the velocity increased with voltage.

The particle movement is assumed to be the effect of viscous friction on the particles from a moving fluid, rather than a force acting on the particle such as dielectrophoresis [18]. Dielectrophoresis always acts toward or away from the electrode edge, but the particles were observed to move past the electrode edge onto the surface. In addition, observation at different heights above the electrodes showed particles moving in convection patterns at high potentials.

At the low and high frequency limits, where the flow velocity was slight, positive dielectrophoresis was observed. The use of the two sizes of particles enabled the effects of dielectrophoresis to be clearly differentiated from fluid driven movement: the difference in the dielectrophoretic 
(a)
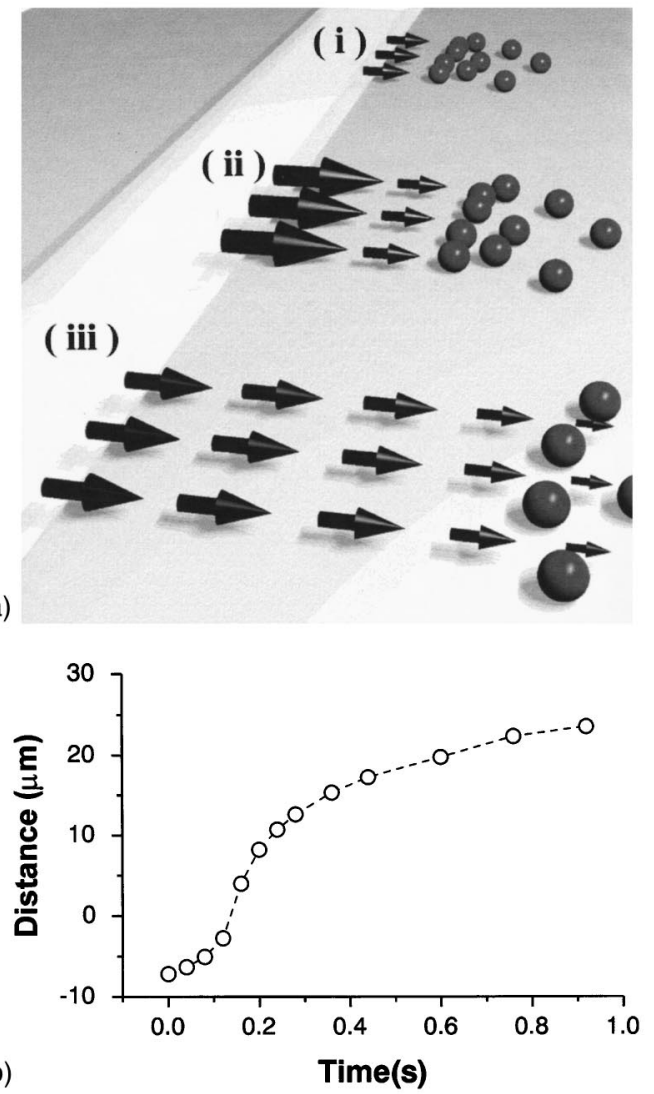
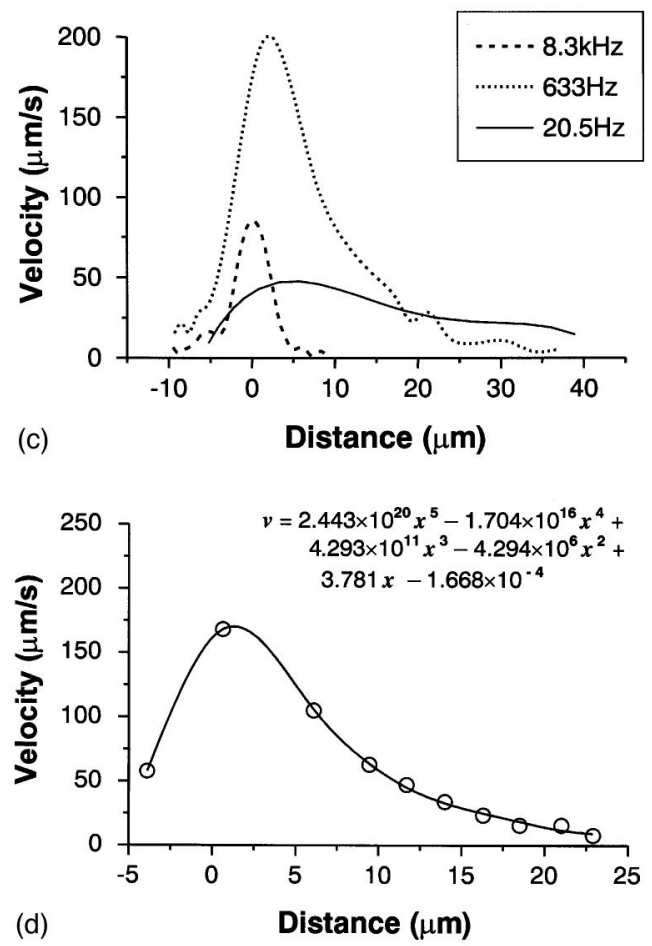

FIG. 3. (a) A schematic diagram of the observed movement of particles on the electrode array. At high frequencies (i), the movement was slight and maximum close to the electrode edge, falling off rapidly with distance. In this case, the particles had a tendency to gather in a band on the electrode surface, which then dispersed much more slowly under the effects of diffusion. At intermediate frequencies (ii), the velocity close to the electrode edge was a maximum and the particles moved rapidly across the electrode surface, continuing from the field of observation. At low frequencies (iii), the velocity at the edge of the electrodes was reduced but the particles continued to move across the field of observation at a significant rate. (b) An experimental graph showing the displacement of a typical particle relative to the electrode edge as a function of time. The experimental conditions were $2 \mathrm{~V}$ peak to peak at $1.01 \mathrm{kHz}$ in electrolyte $A$. (c) An experimental graph of the step averaged velocity against distance from the edge of the electrode, for three frequencies showing the same trends as described for (a). The potential was $2 \mathrm{~V}$ peak to peak with electrolyte $A$. (d) An experimental graph of the step averaged velocity against distance for a single particle in electrolyte $A$ at $2 \mathrm{~V}$ peak to peak and $1.01 \mathrm{kHz}$. The curve is matched with a fifth order polynomial (the equation shown in figure) that accurately describes the behavior of the velocity over the range of interest, in this case 5-20 $\mu \mathrm{m}$.

force magnitude is approximately 6.6 [3], while the velocity of the particle due to the fluid remains constant for the two sizes. The difference in the effects of the two forces allowed them to be separated and the data from frequencies where dielectrophoresis was observed were omitted.

\section{Measurements of fluid velocity}

In order to quantify the fluid velocity, measurements were made of the position of the fluorescent particles on the electrodes as a function of time. The microscope objective was focused on the electrodes, and only particles in focus, i.e., within approximately $1 \mu \mathrm{m}$ of the surface, were used as flow tracers. 557- and 216-nm-diameter particles were observed at three different voltages: 1,2 , and $5 \mathrm{~V}$ peak to peak. For each electrolyte and voltage, the frequency was stepped down from $100 \mathrm{kHz}$, with approximately three points per decade and several minutes of video were recorded at each frequency to allow measurement of several particles.

A typical plot of displacement versus time for one particle is shown in Fig. 3(b) for electrolyte $A$ at $1.01 \mathrm{kHz}$, and $2 \mathrm{~V}$ peak to peak. The displacement plotted is the distance along the $x$ axis [see Fig. 1(b)], with the origin at the electrode edge and the positive direction away from the gap. The average velocity between each point on this graph was then calculated and plotted against the mean position. Figure 3(c) shows velocity curves for particles in electrolyte $A$ at $2 \mathrm{~V}$ peak to peak, and three different frequencies. The trend shown here was similar for each of the three voltages. At high frequencies, the velocity curve was almost symmetrical around the edge of the electrode with a maximum value at the edge, but falling off sharply with distance. In the particular case shown in the figure $(8.3 \mathrm{kHz})$, the velocity is approximately $70 \mu \mathrm{m} / \mathrm{s}$ at the edge. At a distance of $10 \mu \mathrm{m}$ from the edge, the velocity has decreased significantly and is no longer measurable. As the frequency was decreased, the velocity at the edge increased and the distance across the electrode over which the fluid moved also increased. At 633 $\mathrm{Hz}$, the velocity reached a maximum value of approximately $200 \mu \mathrm{m} / \mathrm{s}$ close to the edge, and fell off sharply to a value under $10 \mu \mathrm{m} / \mathrm{s}$ at $30 \mu \mathrm{m}$. Particles were still observed moving at the limit of observation, which was approximately 45 $\mu \mathrm{m}$. As the frequency was decreased further, the velocity 

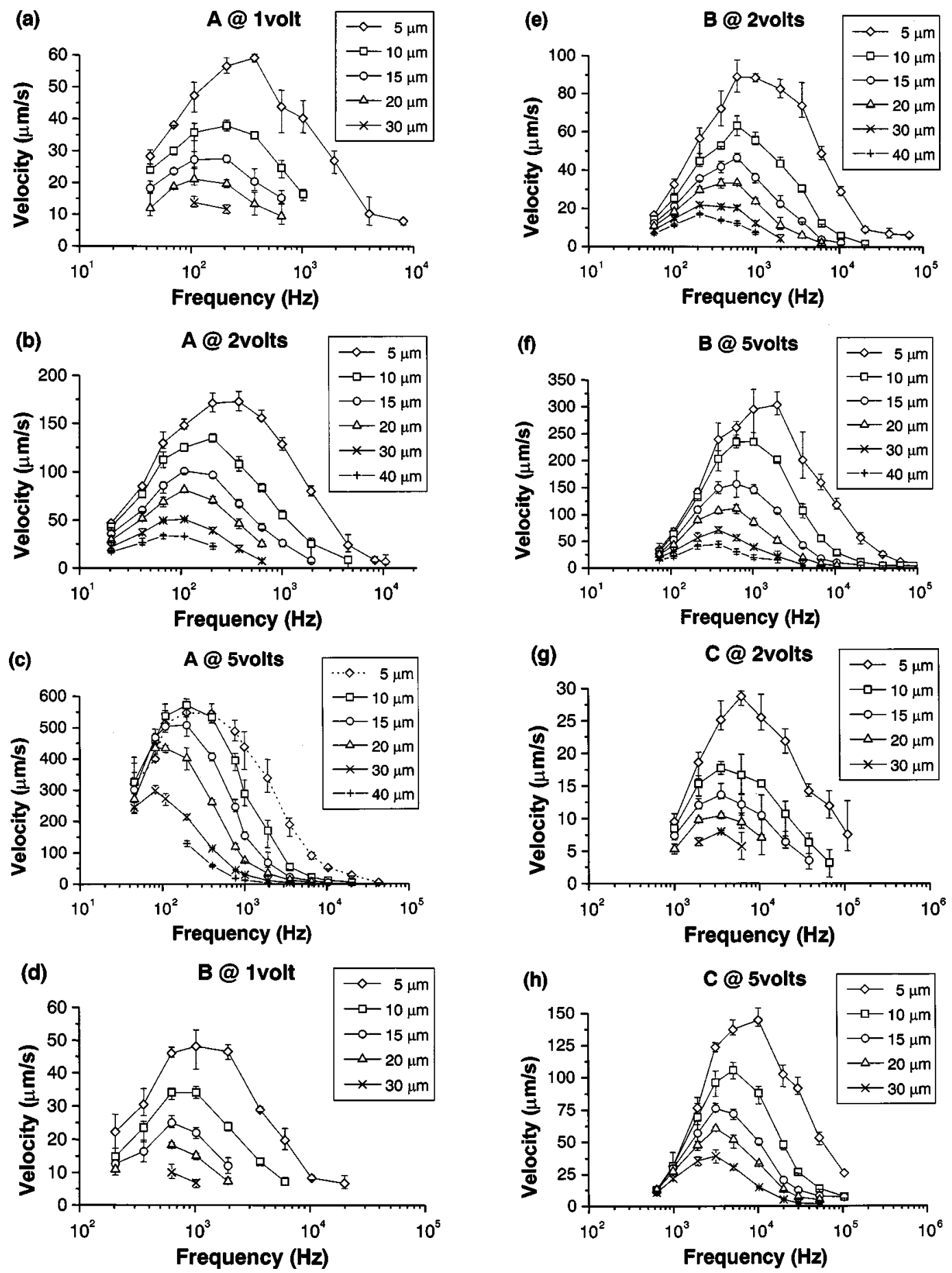

FIG. 4. The experimental data represented as step averaged velocity against frequency for several positions on the electrode surface. (a)-(c) show the results for electrolyte $A$ for voltages of 1,2, and $5 \mathrm{~V}$ peak to peak, respectively. (d)-(f) show the results at the same voltages for electrolyte $B$, and $(\mathrm{g})$ and (h) show the results for electrolyte $C$ at 2 and $5 \mathrm{~V}$ peak to peak.

close to the electrode edge decreased, but the fluid continued to move across the whole electrode surface. At $20.5 \mathrm{~Hz}$, the maximum value was approximately $40-50 \mu \mathrm{m} / \mathrm{s}$, and this maximum value occurred between the edge and a distance of $10 \mu \mathrm{m}$. At $40 \mu \mathrm{m}$, the particle velocity had decreased to approximately $20 \mu \mathrm{m} / \mathrm{s}$.

Each set of velocity data was matched with a best-fit polynomial. Figure 3(d) shows the data for electrolyte $A$ at $2 \mathrm{~V}$ and $1.01 \mathrm{kHz}$, together with a fifth order polynomial matching curve. The values of the velocity at several points: 5,10 ,
15,20 , and $30 \mu \mathrm{m}$, were calculated from these curves. At each point, the average value was calculated and the range of velocities taken to be the uncertainty. Two alternative methods of analyzing the time-displacement data were examined, and a comparison of all three methods is summarized in the Appendix.

The complete set of velocity data is shown in Fig. 4. Figures 4(a)-4(c) show velocity versus frequency curves for electrolyte $A$ at 1,2 , and 5 V. Figures 4(d)-4(f) show the results for electrolyte $B$ for the same potentials and Figs. $4(\mathrm{~g})$ 
and 4(h) show the results for electrolyte $C$ at 2 and $5 \mathrm{~V}$. The velocities calculated using this method for electrolyte $C$ at 1 $\mathrm{V}$ were small with a large uncertainty, and this data set is not presented. In general, the eight graphs show similar behaviors. As frequency decreases the velocity rises to a maximum value and then decreases. The frequency at which the maximum velocity occurs increases with electrolyte conductivity, and decreases with the distance from the edge. For a fixed frequency, the velocity increases with voltage and with decreasing distance. These relationships will be explored further in Sec. IV.

The exception to the general description is Fig. 4(c), which shows the data for electrolyte $A$ at $5 \mathrm{~V}$. Inspection of the curves for the data close to the edge suggests that the velocity has a maximum observed value of approximately $500 \mu \mathrm{m} / \mathrm{s}$. However, at this velocity, the particles will cross a distance of $20 \mu \mathrm{m}$ between consecutive video frames. As a result the analysis method is likely to be too approximate to allow accurate determination of the velocity for experimental points that are only $5 \mu \mathrm{m}$ apart. This issue is discussed more fully in the Appendix.

\section{DISCUSSION}

It has been shown previously that the fluid flow described in this paper cannot be explained in terms of electrothermal effects arising from heating of the fluid by the electric field [12]. The electrothermal force is frequency dependent, with a characteristic frequency $f_{\text {ce }}$ related to the charge relaxation time of the fluid [12], and for the three electrolytes used in this paper $f_{\text {ce }}$ can be calculated to be $500 \mathrm{kHz}$ for electrolyte $A, 2 \mathrm{MHz}$ for electrolyte $B$, and $19 \mathrm{MHz}$ for electrolyte $C$. Below this frequency the magnitude of the electrothermal force rises rapidly over one decade to a maximum value, and then remains constant as the frequency decreases. Taking Fig. 4(a) as an example, it can be seen that the measured velocity starts to rise rapidly at a frequency two orders of magnitude below $f_{\mathrm{ce}}$, and reaches a maximum three orders of magnitude below $f_{\mathrm{ce}}$. The velocity does not remain constant, and an order of magnitude calculation of the expected electrothermally induced velocity [12] gives a value of approximately $1 \mu \mathrm{m} / \mathrm{s}$ at $10 \mu \mathrm{m}$ in from the edge, much less than the observed values. In addition, the velocity magnitude for electrothermal flow is expected to be proportional to $V^{4}$ [12], which again is not observed experimentally [as can be seen from Figs. 5(a) and 5(b)]. Finally, the magnitude of the electrothermal velocity should increase with conductivity but the observed fluid flow velocity decreases. It can therefore be concluded that the observed fluid flow is unlikely to be related to electrothermal effects.

Other authors have also reported movement of particles close to the surface of electrodes $[19,20]$. In these publications, the movement was attributed to fluid flow arising from the interaction of divergent fields around the particles and conduction currents in the double layer. For the type of fluid flow described in this paper, the driving mechanism is related to electrode polarization processes, as has been outlined in previous publications $[14,15]$ and is referred to as ac electro-osmosis. An adequate theory based on this idea remains to be constructed, but there are some qualitative and semiquantitative arguments that can be presented in favor of
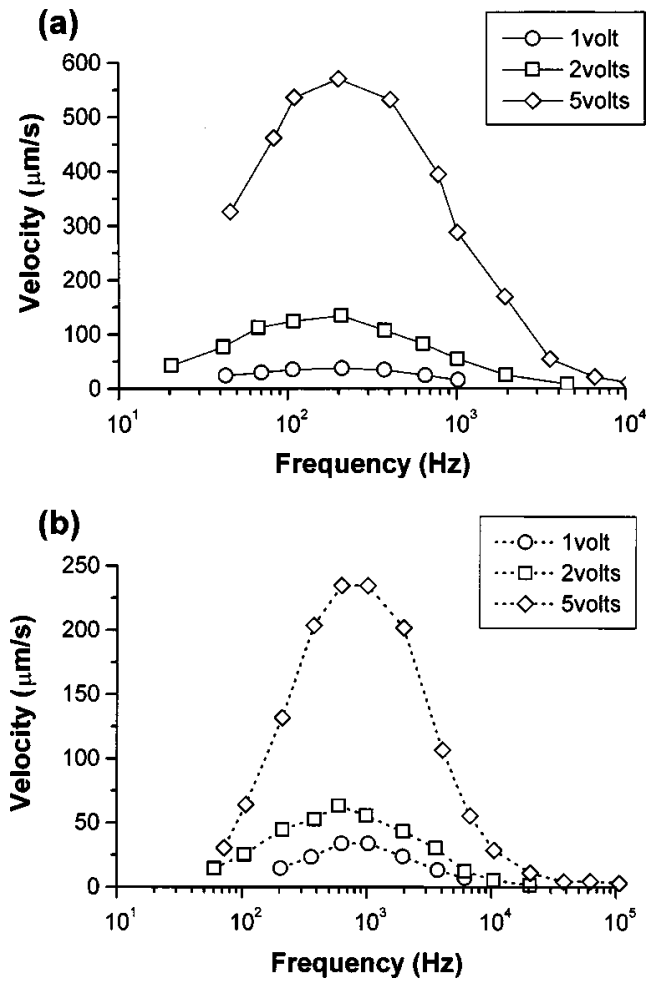

FIG. 5. Comparison of the experimental velocity at $10 \mu \mathrm{m}$ from the edge of the electrode for electrolyte $A$ (a) and $B$ (b) at the three experimental voltages, demonstrating that the three curves have approximately the same frequency of maximum velocity.

the existence of electro-osmosis in divergent ac electric fields.

In the linear approximation, the electro-osmotic velocity is proportional to the charge in the double layer and the tangential electric field [14]. In divergent ac fields the force exerted on the induced charges has a nonzero time average, and acts from the electrode edge across the surface of the electrode, exactly as observed experimentally.

In order to investigate the relationship between experimental measurements of fluid flow and electrode polarization, the method in Ref. [14] was used. Experimental measurements of the electrode impedance were analyzed to give the normalized voltage across the electrolyte and the double layer. Since the measured impedance at high frequencies is the impedance of the bulk electrolyte, the impedance of the double layer can be obtained by assuming a simple series circuit (the values for the voltage drop and impedance represent an average across the entire electrode surface). The voltages across each component can subsequently be derived as a function of frequency. The results of this analysis are shown in Fig. 6 for the three electrolytes, with the real and imaginary parts of the potential across the electrolyte plotted against frequency. The real part [Fig. 6(a)] is minimum at low frequencies, rising sharply to a maximum value at high frequencies. The converse is true for the potential across the double layer. If a simple linear approximation is considered, the ac electro-osmotic velocity is proportional to the product of the tangential electric field and the charge density in the diffuse double layer. Since the potential across the electrolyte is very small at low frequencies, the tangential electric field at the double layer is similarly small. The surface 
(a)

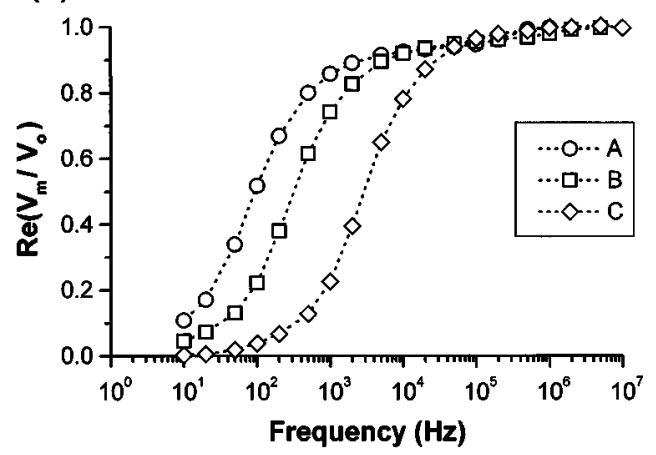

(b)

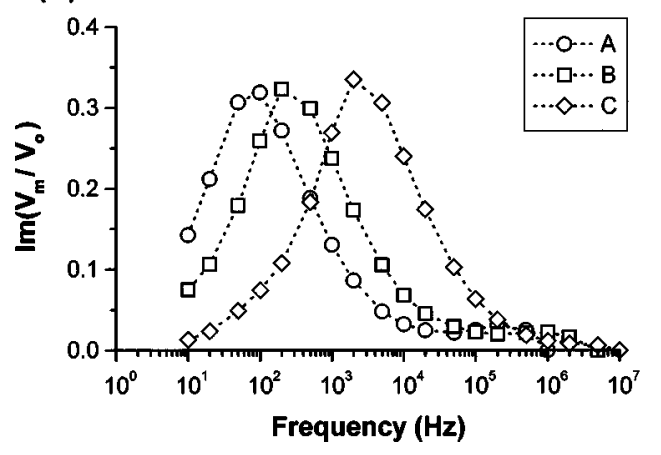

FIG. 6. The normalized voltage across the electrolyte, plotted as real (a) and imaginary (b) parts, calculated from the experimental impedance measurements by modeling the electrolyte and double layers as impedances in series. At low frequencies, the real part of the voltage across the electrolyte is a minimum, and, at high frequencies, the converse is true.

charge density is proportional to the potential across the double layer, and is very small at high frequencies. Therefore, from this simple model, the ac electro-osmotic velocity would be zero at high and low frequency limits and maximum at an intermediate frequency, similar to the experimental observations. This frequency will correlate with the characteristic frequency of the electrode polarization process, as can be seen in Fig. 6(b), where the imaginary component of the potential across the electrolyte is plotted. This figure can be compared with Fig. 7(a), which shows the velocity at 10 $\mu \mathrm{m}$ as a function of frequency for the three electrolytes at 5 $\mathrm{V}$ peak to peak. The frequency of maximum velocity increases with conductivity, and the characteristic frequencies of the velocity and impedance measurements clearly correlate.

These observations provide evidence that ac electroosmosis is responsible for the fluid flow. However, the analysis can be taken further by modeling the double layer as a distributed linear capacitor, as described in Ref. [15]. An equivalent circuit was constructed with the electrolyte described as a concentric series of resistors following semicircular paths between the electrodes, terminated at either end by a discrete component of double-layer capacitance [15]. Each resistor has a different length, and the current polarizes each component of the capacitance at a different time, resulting in a variation in the potential at the double layer/ electrolyte interface across the electrode. The electroosmotic velocity is [15]
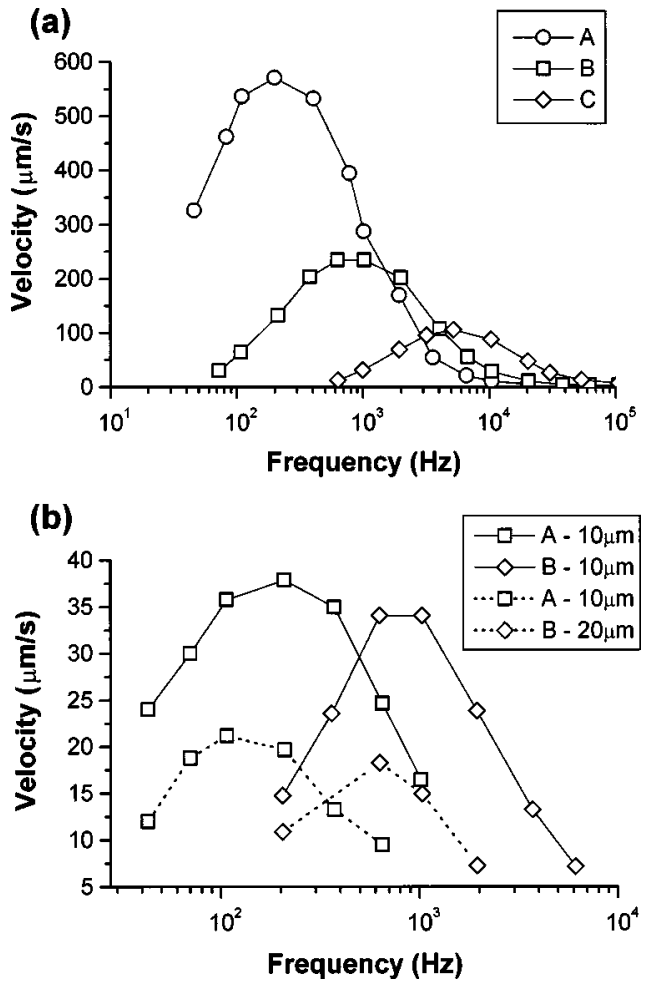

FIG. 7. (a) The experimental velocities at $10 \mu \mathrm{m}$ for $5 \mathrm{~V}$ in the three electrolytes. The frequency of the maximum velocity increases with increasing electrolyte conductivity, but the values of the maximum velocity decreases. (b) The experimental velocities at 10 and $20 \mu \mathrm{m}$ for $1 \mathrm{~V}$ peak to peak in electrolytes $A$ and $B$. The order of magnitude of the velocities for each of the two displacements is the same in both electrolytes.

$$
\langle\nu\rangle=\frac{1}{8} \frac{\varepsilon V_{0}^{2} \Omega^{2}}{\eta x\left(1+\Omega^{2}\right)^{2}},
$$

where $\varepsilon$ is the permittivity of the electrolyte, $V_{0}$ is the potential applied to the electrodes, $\eta$ is the viscosity of the electrolyte, and $x$ is the distance from the center of the gap between the electrodes. The nondimensional frequency $\Omega$ is given by

$$
\Omega=\omega x \frac{\varepsilon}{\sigma} \frac{\pi}{2} \kappa,
$$

where $\omega$ is the angular frequency of the field, $\sigma$ is the conductivity of the electrolyte, and $\kappa$ is the reciprocal Debye length of the double layer. Equation (1) gives a bell-shaped profile for the frequency dependence of the velocity, approaching zero at high and low frequency limits. Figure 8 shows the experimental velocities for electrolytes $A$ (a) and $B$ (b) at $2 \mathrm{~V}$, and electrolyte $C$ at $5 \mathrm{~V}$ (c). For comparison, curves of the theoretical velocity multiplied by a constant correction factor are shown as solid lines. The model predicts symmetrical curves with a maximum at $\Omega=1$ for each of the three electrolytes. As can be seen from Fig. 8, the experimental curves are approximately symmetrical about a value of $\Omega$ of order 1, although the value for the three electrolytes was not the same. This model explains the variation in velocity as a function of frequency and distance, as shown in Figs. 3(a), 3(c), and 4, since the velocity is a function of $\Omega$ 

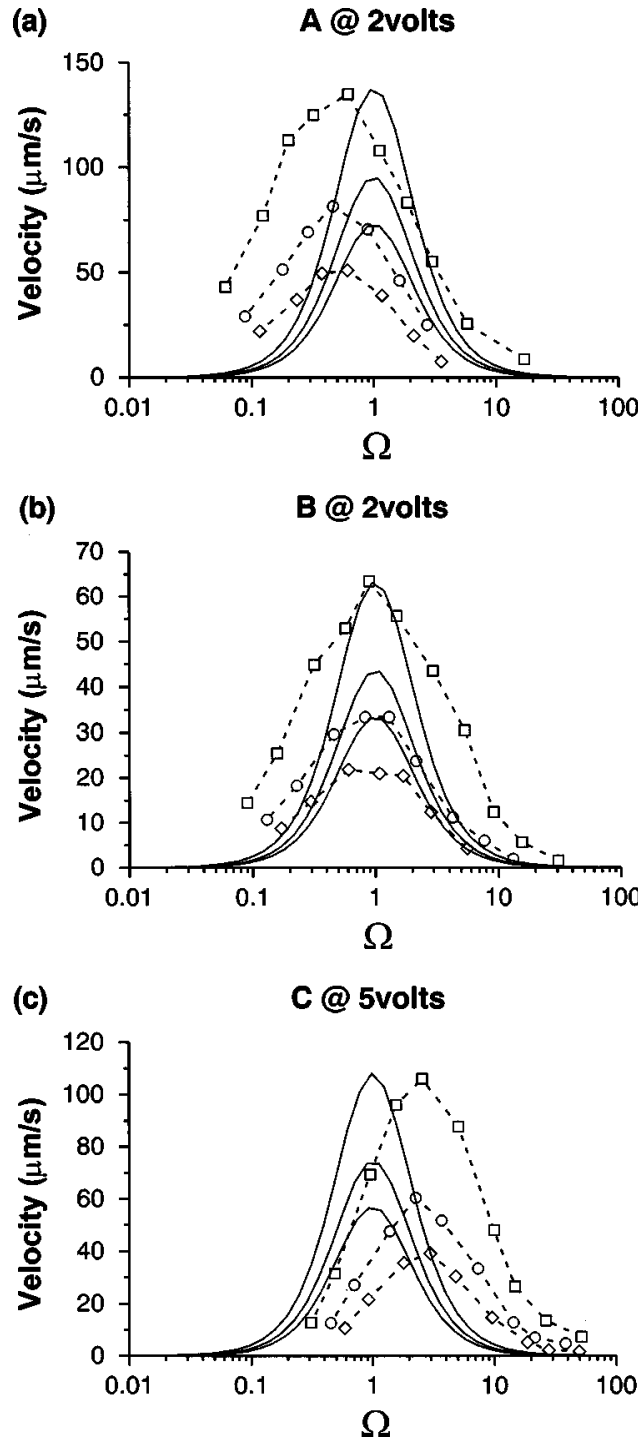

FIG. 8. The experimental velocity data for (a) electrolyte $A$ at 2 $\mathrm{V}$ peak to peak, (b) electrolyte $B$ at $2 \mathrm{~V}$ peak to peak, and (c) electrolyte $C$ at $5 \mathrm{~V}$ peak to peak (dashed lines), plotted against the dimensionless frequency $\Omega$. The theoretical curves (solid lines) are derived from Eq. (1), multiplied by a correction factor. The three lines are for distances from the electrode edge of 10,20, and 30 $\mu \mathrm{m}$. The values for the correction factor were (a) 0.13 , (b) 0.055 , and (c) 0.015 .

rather than simply the frequency $\omega$. The bell-shaped experimental velocity curves are approximately twice as wide as the predicted curve, and are likely to be caused by a spread in relaxation times associated with the geometry and electrode polarization.

The predicted velocity as a function of $\Omega$ is proportional to $1 / x$, but the experimental velocities, although decreasing, do not follow this trend. This is probably due to the fact that the electric field in the experimental electrodes differs from the simple electric field geometry assumed in the model.

Equation (1) also predicts that the velocity remains constant with electrolyte conductivity, contrary to the measured decrease. Figure 7(a) shows the data for $5 \mathrm{~V}$ peak to peak in the three electrolytes, demonstrating the decrease in velocity with increasing electrolyte conductivity. Equation (1) can be matched with the experimental data by multiplying it by a correction factor that varies both with electrolyte conductivity and applied potential. The correction factor is much less than 1 for the higher conductivities, but for electrolyte $A$ the correction factor was of order 0.1 for all three voltages. However, as can be seen in Fig. 7(b), the experimental velocities for $1 \mathrm{~V}$ peak to peak in electrolytes $A$ and $B$ at distances of 10 and $20 \mu \mathrm{m}$ are of the same order of magnitude, and the correction factor was of order 0.1 for both electrolytes. This may be an indication that a theory derived using the linear model for the double layer applies at low values of the potential.

The experimental data are unlikely to be correctly described by the linear approximation outlined in Ref. [15]. In order to account for the observed behavior of the fluid, a more detailed theory must be derived, which includes the Navier-Stokes equation and a better description of the double-layer structure [21].

\section{CONCLUSION}

Detailed experimental measurements have been made of the velocity profile of electrolytes experiencing a force due to an ac electric field on coplanar plate microelectrodes. The measurements have been analyzed as a function of applied signal potential, frequency, electrolyte conductivity, and position on the electrode. It has been found that the fluid velocity on parallel coplanar electrodes always acts in the same direction, from the gap between the electrodes across the electrode surface. The velocity at any position on the surface is zero at low and high frequency limits, with a maximum at a characteristic frequency that does not appear to depend on voltage but does depend on electrolyte conductivity. The measurements indicate that electrothermal effects are not responsible for this type of fluid flow. The experimental velocities can be related to the polarization of the electrodes by a nonuniform electric field. The experimental data have been compared with a simple model based on the interaction of the nonuniform electric field and the induced electrical double layer, a mechanism referred to as ac electro-osmosis. The frequency dependent behavior has been found to be described by the model, but the expected velocities were higher than the experimental by a value that varied with applied potential and electrolyte conductivity. At low potentials, the predicted velocities are in reasonable agreement with the experiments.

In our subsequent paper a more detailed theory will be presented that describes the mechanism of ac electro-osmosis in microelectrodes. A comparison with the experimental data will also be presented.

\section{ACKNOWLEDGMENTS}

The authors would like to acknowledge the European Union for financial support to N. G. G. [Contract No. BIO4CT98-5010 (DG12-SSMI)], the DGES (Spain) (Contract No. PB96-1375), and the Royal Society for financial support to A. R.

\section{APPENDIX: METHODS FOR CURVE MATCHING}

Three methods were examined to determine the best way of obtaining the fluid velocity from the displacement-time measurements. 
(1) The first method involved matching a curve to the displacement vs. time curve [Fig. 3(b)] for each particle. The equation for the best-fit line was then differentiated to give the reciprocal velocity as a function of distance. The values for each particle for a particular position, voltage, frequency, and electrolyte were averaged, and the spread in values taken to be the uncertainty.

(2) The second method involved calculating the average velocity over a step between two positions, and assigning this value to the midpoint of the step. The data for all particles for a particular voltage, frequency, and electrolyte were then plotted against distance. A best-fit curve was then matched to the combined data set, the velocity at a position calculated, and the uncertainty taken to be the maximum spread of values on either side of the curve.

(3) The third method was the one described in the main body of the paper. The average velocity over the step between two points was again calculated and assigned to the midpoint of the step. The velocity was plotted against displacement for each particle, and matched to a best-fit curve. The velocity values for a particular position, voltage, frequency, and electrolyte were then averaged, and the variation in calculated values taken to be the uncertainty.

Figure 9 shows a comparison of the velocity data obtained from each of the three methods for $5 \mathrm{~V}$ peak to peak in electrolyte $C$. The three curves match closely for all of the $2-\mathrm{V}$ data but there are slight differences for the $5-\mathrm{V}$ data. As stated in the main text of the paper, the second and third methods have the drawback that a particle will pass across $\sim 20 \mu \mathrm{m}$ in one frame of the video for the highest velocities $(\sim 500 \mu \mathrm{m} / \mathrm{s})$. Since these two methods average the velocity over the frame, it is not possible to assign an accurate velocity to several points $5 \mu \mathrm{m}$ apart in the step. The first method, on the other hand, extrapolates a curve across the step, and can make an estimate of the behavior. However, this results in large variations in values and a large uncertainty. Large uncertainties were found for the data in general, even when

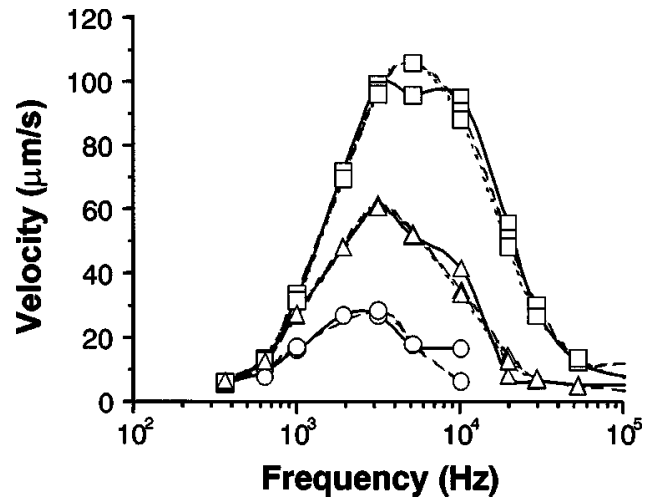

FIG. 9. A comparison of the three methods of curve matching the data for $5 \mathrm{~V}$ peak to peak in electrolyte $C$. Curves for $10 \mu \mathrm{m}$ (squares), $20 \mu \mathrm{m}$ (triangles), and $40 \mu \mathrm{m}$ (circles) are plotted. The solid lines show the results of the first method, the dashed lines those of the second method, and the dotted lines those of the third method. There was no $40-\mu \mathrm{m}$ curve from the third method.

the velocities were not high as can be seen in Fig. 9. The second method also produced large uncertainties since the variation due to Brownian motion, which is small in the displacement data, can be large in the step average velocity data. The third method, for which the curves matched those produced by the second method, eliminates to a certain extent the random variation of Brownian motion for a single particle and produced much smaller values of uncertainty. These smaller values are more likely to be the experimental uncertainty produced for example by the digitizing of the video images. However, since each particle was considered individually, little or no data could be obtained at $40 \mu \mathrm{m}$ with the third method. Although, the velocity data at the higher magnitudes $(5 \mathrm{~V}$ peak to peak in electrolyte $A$, for example) is less likely to be accurate, the third method was chosen. However, it should be noted that for all three methods, the velocity agreed in all cases where the velocities were less than $300 \mu \mathrm{m} / \mathrm{s}$.
[1] R. Pethig, Crit. Rev. Biotech. 16, 331 (1991).

[2] R. Pethig, Y. Huang, X.-B. Wang, and J. P. H. Burt, J. Phys. D 25, 881 (1992).

[3] H. Morgan, M. P. Hughes, and N. G. Green, Biophys. J. 77, 516 (1999).

[4] N. G. Green and H. Morgan, J. Phys. D 30, L41 (1997).

[5] N. G. Green and H. Morgan, J. Phys. D 30, 2626 (1997).

[6] N. G. Green and H. Morgan, J. Phys. Chem. B 103, 41 (1999).

[7] N. G. Green, H. Morgan, and J. J. Milner, J. Biophys. Biochem. Methods 35, 89 (1997).

[8] M. P. Hughes, H. Morgan, F. J. Rixon, J. P. H. Burt, and R. Pethig, Biochim. Biophys. Acta 1425, 119 (1998).

[9] T. Muller, A. Gerardino, T. Schnelle, S. G. Shirley, F. Bordoni, G. De Gasperis, R. Leoni, and G. Fuhr, J. Phys. B 29, 340 (1996).

[10] M. Washizu, S. Suzuki, O. Kurosawa, T. Nishizaka, and T. Shinohara, IEEE Trans. Ind. Appl. 30, 835 (1994).

[11] S. M. Szem, Semiconductor Sensors (Wiley, Somerset, 1994).
[12] A. Ramos, H. Morgan, N. G. Green, and A. Castellanos, J. Phys. D 31, 2338 (1998).

[13] N. G. Green and H. Morgan, J. Phys. D 31, L25 (1998).

[14] A. Ramos, H. Morgan, N. G. Green, and A. Castellanos, J. Electrost. 47, 71 (1999).

[15] A. Ramos, H. Morgan, N. G. Green, and A. Castellanos, J. Colloid Interface Sci. 217, 420 (1999).

[16] Capillary Electrophoresis, edited by P. D. Grossman and J. C. Colburn (Academic, New York, 1992).

[17] H. P. Schwan, Ann. (N.Y.) Acad. Sci. 148, 191 (1968).

[18] H. A. Pohl, Dielectrophoresis (Cambridge University Press, New York, 1978).

[19] M. Trau, D. A. Saville, and I. A. Aksay, Langmuir 13, 6375 (1997).

[20] S.-R. Yeh, M. Seul, and B. I. Shraiman, Nature (London) 386, 57 (1997).

[21] J. Lyklema, Fundamentals of Interface and Colloid Science (Academic, London, 1995). 